\title{
SPLITTING LEAST SQUARES AND COLLOCATION PROCEDURES FOR TWO-POINT BOUNDARY VALUE PROBLEMS
}

\author{
JOHN LOCKER AND P. M. PRENTER ${ }^{1}$
}

(Received 19 October 1983; revised 14 August 1984)

\begin{abstract}
Let $L, T, S$, and $R$ be closed densely defined linear operators from a Hilbert space $X$ into $X$ where $L$ can be factored as $L=T S+R$. The equation $L u=f$ is equivalent to the linear system $T v+R u=f$ and $S u=v$. If $L u=f$ is a two-point boundary value problem, numerical solution of the split system admits cruder approximations than the unsplit equations. This paper develops the theory of such splittings together with the theory of the Methods of Least Squares and of Collocation for the split system. Error estimates in both $L^{2}$ and $L^{\infty}$ norms are obtained for both methods.
\end{abstract}

\section{Introduction}

Let $X$ be a Hilbert space with inner product (, ) and norm \| \|, and let $L, T, S$, and $R$ be closed densely defined linear operators from $X$ into $X$. Let $\mathscr{D}(A), \mathscr{R}(A)$, and $N(A)$ denote the domain, range, and null space, respectively, of an operator $A$. Assume $L$ has the operator splitting or factorization

$$
L=T S+R
$$

where $\mathscr{D}(L)=\{u \in \mathscr{D}(S): S u \in \mathscr{D}(T)\}$ and $\mathscr{D}(S) \subseteq \mathscr{D}(R)$. For a given $f \in X$ we want to consider least squares and collocation methods for approximating solutions to the equation

$$
L u=f \text {, }
$$

which take advantage of the splitting (1.1) by allowing one to work in the subspaces $\mathscr{D}(S)$ and $\mathscr{D}(T)$ rather than in subspaces of $\mathscr{D}(L)$. Our primary goal in

\footnotetext{
${ }^{1}$ Department of Mathematics, Colorado State University, Fort Collins, Colorado 80523, U.S.A. (c) Copyright Australian Mathematical Society 1985, Serial-fee code 0334-2700/85
} 
this paper will be the theory and applicability of such least squares and collocation splitting schemes to higher order linear two-point boundary value problems in the Hilbert space $X=L^{2}[a, b]$.

The starting point of our splitting method is to replace equation (1.2) by the equivalent system of equations

$$
\begin{gathered}
T v+R u=f \\
S u=v .
\end{gathered}
$$

For simplicity we assume that $L$ is 1-1 onto $X$ and that $S$ is 1-1. Clearly $u \in \mathscr{D}(L)$ solves (1.2) iff the pair $(v, u) \in \mathscr{D}(T) \times \mathscr{D}(S)$ solves (1.3) and (1.4).

There is a considerable literature on numerical procedures for systems of two-point boundary value problems with $[1,4,5,6,11,14,21]$ treating collocation for systems. Moreover, splitting procedures are used in a number of codes including the Keller box scheme [14] and the de Boor-Weiss code [6] for systems of two-point boundary value problems. The procedures of this paper are considerably more flexible than other extant procedures and allow a great deal of latitude in selection of approximating subspaces. Moreover, the theory and methodology carry over to partial differential equations (PDE) in such a way as to offer highly efficient alternatives to extant methods for important higher order PDE such as the biharmonic equation (see $[3,7,8,9,10,24]$ for examples of schemes requiring alternating iterations of the split operator). These generalizations will appear in forthcoming research of the second author for which this paper lays a mathematical foundation.

\section{The method of splitting least squares}

The simplest vantage from which to devise a split least squares procedure for solving (1.2) via the linked system (1.3) and (1.4) is to introduce the product space $Y=X \times X$ and define the linear operator $K$ from $\mathscr{D}(T) \times \mathscr{D}(S)=\mathscr{X}$ into $Y$ by

$$
K z=(R u+T v, S u-v), \quad z=(v, u) \in \mathscr{X} .
$$

Let $Y$ carry the natural product, inner product and norm structure

$$
\left\langle z_{1}, z_{2}\right\rangle=\left(v_{1}, v_{2}\right)+\left(u_{1}, u_{2}\right), \quad|z|=\sqrt{\langle z, z\rangle},
$$

for all $z_{1}=\left(v_{1}, u_{1}\right)$ and $z_{2}=\left(v_{2}, u_{2}\right)$ in $Y$.

For the given $f \in X$ set

$$
y=(f, 0)
$$

Then

$$
|K z-y|^{2}=\|R u+T v-f\|^{2}+\|S u-v\|^{2},
$$


where (2.2) defines the functional

$$
J(z)=\|R u+T v-f\|^{2}+\|S u-v\|^{2}=|K z-y|^{2}
$$

on $\mathscr{X}$. It is clear that the minimum value of $J$ is 0 and the minimum is achieved at $(v, u) \in \mathscr{X}$ where $u$ is the unique solution to $L u=f$ with $S u=v$.

\section{THEOREM 2.1. $K$ is $1-1$.}

Proof. If $K(v, u)=0$, then $R u+T v=0$ and $S u=v$. It follows that $u \in$ $\mathscr{D}(T S)=\mathscr{D}(L)$ with $L u=(T S+R) u=0$. Since $L$ is $1-1, u=0$ and $v=S u=0$. Q.E.D.

The method of Splitting Least Squares (SLS) applied to the equation $L u=f$ comprises choosing finite dimensional subspaces $\mathscr{S}_{u} \subseteq \mathscr{D}(S)$ and $\mathscr{S}_{v} \subseteq \mathscr{D}(T)$ and finding $\tilde{z}=(\tilde{v}, \tilde{u}) \in \mathscr{S}=\mathscr{S}_{v} \times \mathscr{S}_{u}$ solving the minimization problem

$$
J(\tilde{z})=\min _{w \in \mathscr{S}} J(w)
$$

or equivalently,

$$
|K \tilde{z}-y|=\min _{w \in \mathscr{S}}|K w-y|, \quad y=(f, 0) .
$$

Existence and uniqueness of $\tilde{z}$ are guaranteed by the finite-dimensionality of $\mathscr{S}$ and by $K$ being 1-1.

Clearly $\tilde{z}$ solves (2.4) iff

$$
\langle K \tilde{z}-y, K \xi\rangle=0 \text { for all } \xi=(\phi, \psi) \in \mathscr{S} .
$$

It follows that $\tilde{z}=(\tilde{v}, \tilde{u})$ satisfies the linked pair of equations

$$
\begin{aligned}
& (R \tilde{u}+T \tilde{v}-f, T \phi)=(S \tilde{u}-\tilde{v}, \phi) \quad \text { for all } \phi \in \mathscr{S}_{v}, \\
& (S \tilde{u}-\tilde{v}, S \psi)=(f-R \tilde{u}-T \tilde{v}, R \psi) \quad \text { for all } \psi \in \mathscr{S}_{u} .
\end{aligned}
$$

For

$$
\begin{aligned}
& \mathscr{S}_{u}=\operatorname{span}\left\{\psi_{1}, \psi_{2}, \ldots, \psi_{N}\right\}, \\
& \mathscr{S}_{v}=\operatorname{span}\left\{\phi_{1}, \phi_{2}, \ldots, \phi_{M}\right\}
\end{aligned}
$$

let

$$
\tilde{u}=\sum_{k=1}^{N} u_{k} \psi_{k} \text { and } \tilde{v}=\sum_{j=1}^{M} v_{j} \phi_{j}
$$

Equation (2.6) yields the SLS algorithm

$$
\begin{array}{ll}
\sum_{j=1}^{M} a_{i j} v_{j}+\sum_{k=1}^{N} b_{i k} u_{k}=\left(f, T \phi_{i}\right), & 1 \leqslant i \leqslant M, \\
\sum_{j=1}^{M} c_{i j} v_{j}+\sum_{k=1}^{N} d_{i k} u_{k}=\left(f, R \psi_{i}\right), & 1 \leqslant i \leqslant N,
\end{array}
$$


where

$$
\begin{aligned}
a_{i j} & =\left(T \phi_{i}, T \phi_{j}\right)+\left(\phi_{i}, \phi_{j}\right), \\
b_{t k} & =\left(T \phi_{i}, R \psi_{k}\right)-\left(\phi_{i}, S \psi_{k}\right), \\
c_{i j} & =-\left(S \psi_{i}, \phi_{j}\right)+\left(R \psi_{i}, T \phi_{j}\right), \\
d_{i k} & =\left(S \psi_{l}, S \psi_{k}\right)+\left(R \psi_{l}, R \psi_{k}\right) .
\end{aligned}
$$

Choosing $\left\{\phi_{j}: 1 \leqslant j \leqslant M\right\}$ and $\left\{\psi_{k}: 1 \leqslant k \leqslant N\right\}$ linearly independent subsets of $\mathscr{D}(T)$ and $\mathscr{D}(S)$, respectively, leads to a uniquely solvable linked system of $M+N$ equations in $M+N$ unknowns.

In applications to two-point boundary value problems, we work in $X=L^{2}[a, b]$ with the usual $L^{2}$ inner product and norm

$$
(u, v)=\int_{a}^{b} u v, \quad\|u\|=\sqrt{(u, u)} .
$$

The subspaces $\mathscr{S}_{v}$ and $\mathscr{S}_{u}$ can be chosen in a variety of ways. For purposes of concise error estimation and sparcity of the matrices associated with the SLS computations, we shall choose $\mathscr{S}_{v}$ and $\mathscr{S}_{u}$ to be spaces of Hermite splines. Specifically, let $\Delta: a=s_{0}<s_{1}<\cdots<s_{N}=b$ be a partition of $[a, b]$. Let

$$
\mathrm{Sp}(m, \Delta, k)=\left\{u \in C^{k}[a, b]: u \in P_{m}\left[I_{l}\right], 1 \leqslant i \leqslant N\right\}
$$

denote the space of polynomial splines of smoothness class $k$ which reduce to polynomials of degree $m$ on each subinterval $I_{t}=\left[s_{t-1}, s_{t}\right]$ of the partition $\Delta$. Let $h=\max \left\{s_{i}-s_{i-1}: 1 \leqslant i \leqslant N\right\}$ and $h_{-}=\min \left\{s_{i}-s_{i-1}: 1 \leqslant i \leqslant N\right\}$ denote the maximum and minimum mesh grading of $\Delta$. We assume that $\Delta=\Delta(N)$ is selected from a family $\mathscr{M}$ of uniformly graded meshes, i.e., $\Delta \in \mathscr{M}$ implies there exists a constant $\sigma$ independent of $\Delta$ such that $h / h_{-} \leqslant \sigma$. The partition $\Delta$ gives rise to two families of functions on which error estimates are available for $\mathrm{Sp}(m, \Delta, k)$. In particular, we introduce the Sobolev space

$$
H^{n}[a, b]=\left\{\begin{array}{ll}
u \in C^{n-1}[a, b]: & u^{(n-1)} \text { is absolutely continuous on }[a, b] \\
\text { and } u^{(n)} \in L^{2}[a, b]
\end{array}\right\}
$$

with the Sobolev norm

$$
\|u\|_{n}=\left[\sum_{j=0}^{n}\left\|u^{(\jmath)}\right\|^{2}\right]^{1 / 2},
$$

the space

$$
H_{\Delta}^{n, k}[a, b]=\left\{u \in H^{n}[a, b]: u \in H^{n+k}\left[I_{i}\right], 1 \leqslant i \leqslant N\right\}
$$

with the norm

$$
\|u\|_{n, k}=\left[\sum_{i=1}^{N}\|u\|_{H^{n+k}\left[I_{i}\right]}^{2}\right]^{1 / 2},
$$


and the space

$$
C_{\Delta}^{n, k}[a, b]=\left\{u \in H^{n}[a, b]: u \in C^{n+k}\left[I_{i}\right], 1 \leqslant i \leqslant N\right\}
$$

with the norm

$$
\|u\|_{n, k}=\max _{0 \leqslant j \leqslant n+k}\left\|u^{(j)}\right\|_{\infty},
$$

where \|\|$_{\infty}$ is the $L^{\infty}[a, b]$ norm. Let $H_{\Delta}^{k}[a, b]=H_{\Delta}^{0, k}[a, b]$ and $C_{\Delta}^{k}[a, b]=$ $C_{\Delta}^{0, k}[a, b]$.

For each $u \in C^{n-1}[a, b]$ it is well known that there exists a unique $\bar{u} \in$ $\operatorname{Sp}(2 n-1, \Delta, n-1)$ satisfying

$$
\bar{u}^{(J)}\left(s_{i}\right)=u^{(j)}\left(s_{\imath}\right) \text { for } 0 \leqslant i \leqslant N \text { and } 0 \leqslant j \leqslant n-1 .
$$

The function $\bar{u}$ is the piecewise Hermite interpolate of degree $2 n-1$ to $u$, or more briefly, the interpolate to $u$ from $\operatorname{Sp}(2 n-1, \Delta, n-1)$. The following theorems are proved in a variety of places (see [16]).

THEOREM 2.2. If $u \in H_{\Delta}^{n, k}[a, b]$ where $0 \leqslant k \leqslant n$, then

$$
\left\|u^{(j)}-\bar{u}^{(j)}\right\| \leqslant \gamma\left\|u^{(n+k)}\right\| h^{n+k-j} \text { for } 0 \leqslant j \leqslant n+k
$$

and

$$
\left\|u^{(j)}-\bar{u}^{(j)}\right\|_{\infty} \leqslant \gamma\left\|u^{(n+k)}\right\| h^{n+k-j-1 / 2} \text { for } 0 \leqslant j \leqslant n+k-1
$$

THEOREM 2.3. If $u \in C_{\Delta}^{n, k}[a, b]$ where $0 \leqslant k \leqslant n$, then

$$
\left\|u^{(j)}-\bar{u}^{(j)}\right\|_{\infty} \leqslant \gamma\left\|u^{(n+k)}\right\|_{\infty} h^{n+k-j} \text { for } 0 \leqslant j \leqslant n+k .
$$

\section{Mathematical preliminaries}

Analysis of convergence of SLS and split collocation approximates to the split system of differential equations (1.3) and (1.4) is considerably simplified if one has in hand the Green's matrix associated with the split system. In this section we work in an arbitrary Hilbert space $X$ and prove that the embedding operator $K$ is onto $Y=X \times X$ whenever $L, T$, and $S$ are Fredholm operators. This information is specialized in Section 4 to the case of differential operators in $L^{2}[a, b]$ to show $K$ has a bounded inverse and to obtain the Green's matrix for (1.3) and (1.4) together with the regularity and boundedness properties of the components of this matrix.

A linear operator $Q$ from $X$ into $X$ is said to be a Fredholm operator if $Q$ is a closed densely defined linear operator with closed range for which $\operatorname{dim} N(Q)<\infty$ and $\operatorname{dim} N\left(Q^{*}\right)<\infty$ where $Q^{*}$ is the Hilbert space adjoint of $Q$. Assume $L, T$, 
and $S$ are all Fredholm operators. Then TS is also a Fredholm operator in $X$ with $\mathscr{D}(T S)=\mathscr{D}(L)$.

Let $S^{\dagger}=S^{-1} P_{S}$ where $P_{S}$ is the orthogonal projection of $X$ onto $\mathscr{R}(S)$. Note that $S^{\dagger} S u=u$ for all $u \in \mathscr{D}(S)$ since $S$ is 1-1. Let

$$
\tau=T+R S^{\dagger}
$$

with $\mathscr{D}(\tau)=\mathscr{D}(T)$.

LEMMA 3.1. $\mathscr{R}(S) \cap N(\tau)=\{0\}$.

Proof. Suppose $\phi \in \mathscr{R}(S) \cap N(\tau)$. Then $\phi=S u$ for some $u \in \mathscr{D}(S)$ and $\tau \phi=0$. Hence,

$$
\begin{aligned}
0 & =\tau \phi \\
& =\tau S u \\
& =\left(T+R S^{\dagger}\right) S u \\
& =T S u+R u \\
& =L u .
\end{aligned}
$$

Since $L$ is $1-1, u=0$ and $\phi=0$. Q.E.D.

A linear operator $M$ in $X$ with $\mathscr{D}(T) \subseteq \mathscr{D}(M) \subseteq X$ is said to be $T$-compact if $M \mid \mathscr{D}(T)$ is compact from $\mathscr{D}(T)$ under the $T$-graph norm into $X$. It is well known that if $M$ is $T$-compact with $\mathscr{D}(T) \subseteq \mathscr{D}(M)$, then $T+M$ is a Fredholm operator in $X$. Henceforth, we assume

$$
R S^{\dagger} \text { is } T \text {-compact, and } R S^{\dagger} \text { is continuous on } X \text {. }
$$

LEMMA 3.2. If $R S^{\dagger}$ is $T$-compact, then $\tau$ is a Fredholm operator in $X$.

Proof. See Schechter [23, page 168].

LEMMA 3.3. $K$ has closed range in $X \times X$.

Proof. Let $\left(v_{t}, u_{t}\right), i=1,2, \ldots$, be a sequence in $\mathscr{D}(T) \times \mathscr{D}(S)$ with

$$
K\left(v_{t}, u_{t}\right)=\left(\xi_{i}, \eta_{t}\right) \rightarrow(\xi, \eta) .
$$

Then

$$
\begin{gathered}
R u_{\imath}+T v_{\imath}=\xi_{\imath} \rightarrow \xi, \\
S u_{\imath}-v_{i}=\eta_{\imath} \rightarrow \eta .
\end{gathered}
$$

Applying $R S^{\dagger}$ to (3.4), we obtain

$$
R u_{i}-R S^{\dagger} v_{i}=R S^{\dagger} \eta_{i} \rightarrow R S^{\dagger} \eta
$$


Subtracting this from (3.3) we get

$$
\left(T+R S^{\dagger}\right) v_{i}=\tau v_{t}=\xi_{i}-R S^{\dagger} \eta_{i} \rightarrow \xi-R S^{\dagger} \eta .
$$

Setting

$$
\psi_{1}=\xi_{i}-R S^{\dagger} \eta_{1}, \psi=\xi-R S^{\dagger} \eta,
$$

we arrive at the upper triangular system

$$
\begin{gathered}
S u_{t}-v_{t}=\eta_{t} \rightarrow \eta, \\
\tau v_{t}=\psi_{i} \rightarrow \psi .
\end{gathered}
$$

For $i=1,2, \ldots$ let

$$
v_{\imath}=w_{\imath}+z_{\imath}
$$

where $w_{\imath} \in \mathscr{D}(\tau) \cap N(\tau)^{\perp}$ and $z_{i} \in N(\tau)$. From (3.6)

$$
\tau^{\dagger} \tau v_{1}=\tau^{\dagger} \tau w_{1}=w_{i}=\tau^{\dagger} \psi_{i} \rightarrow \tau^{\dagger} \psi=w,
$$

where $\tau^{\dagger}$ is the Moore-Penrose generalized inverse of $\tau$. Thus, $w$ and $w_{i} \in \mathscr{D}(\tau) \cap$ $N(\tau)^{\perp}$. Noting that $w=\tau^{\dagger} \psi=\tau^{\dagger} \xi-\tau^{\dagger} R S^{\dagger} \eta$, we have

$$
S u_{t}-z_{i}=S u_{i}-\left(v_{i}-w_{\imath}\right)=\eta_{t}+w_{i} \rightarrow \eta+w
$$

with $S u_{i} \in \mathscr{R}(S)$ and $z_{i} \in N(\tau)$ for $i=1,2, \ldots$

But $\operatorname{dim} N(\tau)<\infty$ since $\tau$ is a Fredholm operator. It follows from Lemma 3.1 that $\mathscr{R}(S)+N(\tau)$ is closed in $X$ and that $\mathscr{R}(S) \oplus N(\tau)$ is a topological direct sum. Thus, the associated projection operators from $\mathscr{R}(S) \oplus N(\tau)$ onto $\mathscr{R}(S)$ and $N(\tau)$ are continuous, and from (3.8) we obtain

$$
S u_{\imath} \rightarrow S u \text { for some } u \in \mathscr{D}(S)
$$

and

$$
z_{i} \rightarrow z \in N(\tau)
$$

But (3.7) gave $w_{1} \rightarrow w$ with $w_{i}$ and $w$ in $\mathscr{D}(\tau) \cap N(\tau)^{\perp}$. Thus,

$$
v_{t}=w_{i}+z_{\imath} \rightarrow w+z=v
$$

where $v \in \mathscr{D}(\tau)=\mathscr{D}(T)$. Summarizing, we have $v_{i} \rightarrow v, \tau v_{i}=\psi_{i} \rightarrow \psi$, and $S u_{i}-$ $v_{i} \rightarrow S u-v=\eta$. Since $\tau$ is closed, $v \in \mathscr{D}(\tau)$ and $\tau v=\psi$. Thus,

$$
S u-v=\eta
$$

and

$$
\tau v=\left(T+R S^{\dagger}\right) v=\psi=\xi-R S^{\dagger} \eta .
$$

Applying $R S^{\dagger}$ to (3.9) gives

$$
R u-R S^{\dagger} v=R S^{\dagger} \eta
$$

Rewriting (3.10) as

$$
R u+T v+R S^{\dagger} v-R u=\xi-R S^{\dagger} \eta
$$


and adding these two equations yields

$$
R u+T v=\xi .
$$

Equations (3.9) and (3.11) yield

$$
K(v, u)=(\xi, \eta) \in \mathscr{R}(K) .
$$

\section{LEMMA 3.4. $K$ is a closed linear onerntor in $X \times X$.}

Proof. Let $\left(v_{i}, u_{i}\right), i=1,2, \ldots$, be a sequence in $\mathscr{D}(K)=\mathscr{D}(T) \times \mathscr{D}(S)$ with $\left(v_{t}, u_{t}\right) \rightarrow(v, u)$ and $K\left(v_{i}, u_{t}\right)=\left(\xi_{i}, \eta_{t}\right) \rightarrow(\xi, \eta)$. Following the proof of Lemma 3.3, there is $u_{0} \in \mathscr{D}(S)$ and $v_{0} \in \mathscr{D}(T)$ with

$$
K\left(v_{0}, u_{0}\right)=\left(R u_{0}+T v_{0}, S u_{0}-v_{0}\right)=(\xi, \eta),
$$

and $v_{i} \rightarrow v_{0}$ and $S u_{i} \rightarrow S u_{0}$. Thus, $v=v_{0} \in \mathscr{D}(T), u \in \mathscr{D}(S)$ with $S u=S u_{0}$, and $u=u_{0}$ since $S$ is 1-1. Therefore, $(v, u)=\left(v_{0}, u_{0}\right) \in \mathscr{D}(K)$ and $K(v, u)=(\xi, \eta)$. Q.E.D.

TheOREM 3.5. $\mathscr{R}(K)=X \times X$.

Proof. We have shown that $K$ is a closed densely defined linear operator in $X \times X$ having closed range. Thus, to establish the theorem it suffices to show that $N\left(K^{*}\right)=\{0\}$. Take any $(x, y) \in N\left(K^{*}\right)$. Then

$$
\begin{aligned}
0 & =\langle K(v, u),(x, y)\rangle=\langle(R u+T v, S u-v),(x, y)\rangle \\
& =(R u, x)+(T v, x)+(S u, y)-(v, y)
\end{aligned}
$$

for all $u \in \mathscr{D}(S)$ and all $v \in \mathscr{D}(T)$. Setting $u=0$ in this expression, we get

$$
(T v, x)=(v, y)
$$

for all $v \in \mathscr{D}(T)$. Hence $x \in \mathscr{D}\left(T^{*}\right)$ with $T^{*} x=y$. Similarly, setting $v=0$ in (3.12), we have

$$
(R u, x)+\left(S u, T^{*} x\right)=0
$$

for all $u \in \mathscr{D}(S)$. Thus, for any $u \in \mathscr{D}(L)=\mathscr{D}(T S) \subseteq \mathscr{D}(S)$ we have

$$
(L u, x)=(T S u+R u, x)=\left(S u, T^{*} x\right)+(R u, x)=0 .
$$

Since $\mathscr{R}(L)=X$, we conclude that $x=0$ and $y=T^{*} x=0$. It follows that $N\left(K^{*}\right)=\{0\}$ and $\mathscr{R}(K)=X \times X$. Q.E.D.

\section{Differential operators and the Green's matrix}

We now specialize the methods of the previous sections to differential operators. Specifically, let $L, T$, and $S$ denote linear differential operators in $L^{2}[a, b]$ of orders $m+n, m$, and $n$, respectively, with $m \leqslant n$. Let $R$ denote a differential 
operator in $L^{2}[a, b]$ of order $p \leqslant n$ with $\mathscr{D}(S) \subseteq \mathscr{D}(R)$. Clearly these differential operators are all Fredholm operators in $L^{2}[a, b]$. Assume $L$ is $1-1$ with $\mathscr{R}(L)=$ $L^{2}[a, b]$, and assume

$$
L=T S+R
$$

where $S$ is 1-1. It follows that $\mathscr{D}(L)=\mathscr{D}(T S), R S^{\dagger}$ is $T$-compact, $\tau=T+R S^{\dagger}$ is Fredholm, and $R S^{\dagger}$ is continuous on $L^{2}[a, b]$. Thus, the theory of Section 3 obtains, and the linear operator

$$
K: \mathscr{D}(T) \times \mathscr{D}(S) \subseteq L^{2}[a, b] \times L^{2}[a, b] \rightarrow L^{2}[a, b] \times L^{2}[a, b]
$$

given by

$$
K(v, u)=(R u+T v, S u-v)
$$

is closed, densely defined, $1-1$, and onto $L^{2}[a, b] \times L^{2}[a, b]$.

For the Sobolev space $H^{n}[a, b]$ we have the norm

$$
\|u\|_{n}=\left[\sum_{J=0}^{n}\left\|u^{(J)}\right\|^{2}\right]^{1 / 2}
$$

introduced in Section 2, as well as the equivalent norm

$$
\|u\|_{H^{n}[a, b]}=\sum_{j=0}^{n-1}\left\|u^{(j)}\right\|_{\infty}+\left\|u^{(n)}\right\| .
$$

Let $Y=L^{2}[a, b] \times L^{2}[a, b]$ carry the natural product $L^{2}$ structure introduced in Section 2, and let $\mathscr{D}(K)=\mathscr{D}(T) \times \mathscr{D}(S)$ carry the product Sobolev topology induced by the norm

$$
\|(v, u)\|=\|v\|_{m}+\|u\|_{n} .
$$

Then $\mathscr{D}(K)$ is a Banach space under the product Sobolev topology, $K$ is continuous from $\mathscr{D}(K)$ under the product Sobolev topology into $Y$ under the product $L^{2}$ topology, and $K^{-1}$ is continuous from $Y$ under the product $L^{2}$ topology into $\mathscr{D}(K)$ under the product Sobolev topology. Thus,

and

$$
|K(v, u)| \leqslant \gamma\|\|(v, u) \|, \quad(v, u) \in \mathscr{D}(K),
$$

$$
\left\|K^{-1} y\right\| \leqslant\left\|K^{-1}\right\||y|, \quad y \in Y .
$$

For each $s \in[a, b]$, each $k=0,1, \ldots, m-1$, and each $j=0,1, \ldots, n-1$ we define linear functionals

$$
\lambda_{k s}^{v}: Y \rightarrow \mathbf{R}, \quad \lambda_{j s}^{u}: Y \rightarrow \mathbf{R},
$$

through the equations

$$
\begin{aligned}
& v^{(k)}(s)=\lambda_{k s}^{v} y=\frac{d^{k}}{d s^{k}} P_{1} K^{-1} y(s), \\
& u^{(j)}(s)=\lambda_{j s}^{u} y=\frac{d^{J}}{d s^{j}} P_{2} K^{-1} y(s),
\end{aligned}
$$


where

$$
\left\{\begin{array} { l } 
{ P _ { 1 } : X \times X \rightarrow X } \\
{ P _ { 1 } ( v , u ) = v }
\end{array} \text { and } \quad \left\{\begin{array}{l}
P_{2}: X \times X \rightarrow X \\
P_{2}(v, u)=u
\end{array}\right.\right.
$$

are the usual coordinate projectors. It follows that if $\mathscr{D}(T)$ and $\mathscr{D}(S)$ carry their $H^{m}$ and $H^{n}$ Sobolev topologies, respectively, then $\lambda_{k s}^{v}$ and $\lambda_{j s}^{w}$ are both continuous linear functionals on $Y$ with its product $L^{2}$ iopology. As such $\lambda_{k s}$ and $\lambda_{j s}^{*}$ have representors or generalized Green's functions

$$
\mathscr{G}_{k s}^{v}=\left(\mathscr{G}_{k s}^{v 1}, \mathscr{G}_{k s}^{v 2}\right) \in X \times X \text { and } \mathscr{G}_{j s}^{u}=\left(\mathscr{G}_{j s}^{u 1}, \mathscr{G}_{j s}^{u 2}\right) \in X \times X
$$

satisfying

$$
\left.\begin{array}{l}
v^{(k)}(s)=\left\langle\mathscr{G}_{k s}^{v}, y\right\rangle, \\
u^{(J)}(s)=\left\langle\mathscr{G}_{j s}^{u}, y\right\rangle,
\end{array}\right\}
$$

for $y \in Y$ and $K^{-1} y=(v, u) \in \mathscr{D}(K)=\mathscr{D}(T) \times \mathscr{D}(S)$.

Choose

$$
g_{k s}^{v}=\left(g_{k s}^{v 1}, g_{k s}^{v 2}\right) \in \mathscr{D}(K) \text { and } g_{j s}^{u}=\left(g_{j s}^{u 1}, g_{j s}^{u 2}\right) \in \mathscr{D}(K)
$$

such that

$$
\left.\begin{array}{l}
K g_{k s}^{v}=\mathscr{G}_{j s}^{v}=\left(R g_{k s}^{v 2}+T g_{k s}^{v 1}, S g_{k s}^{v 2}-g_{k s}^{v 1}\right) \\
K g_{j s}^{u}=\mathscr{G}_{j s}^{u}=\left(R g_{j s}^{u 2}+T g_{j s}^{u 1}, S g_{j s}^{u 2}-g_{j s}^{u 1}\right)
\end{array}\right\}
$$

Thus,

$$
\left.\begin{array}{l}
R g_{k s}^{v 2}+T g_{k s}^{v 1}=\mathscr{G}_{k s}^{v 1}, \\
S g_{k s}^{v 2}-g_{k s}^{v 1}=\mathscr{G}_{k s}^{v 2},
\end{array}\right\}
$$

and

$$
\left.\begin{array}{l}
R g_{j s}^{u 2}+T g_{j s}^{u 1}=\mathscr{G}_{j s}^{u 1}, \\
S g_{j s}^{u 2}-g_{j s}^{u 1}=\mathscr{G}_{j s}^{u 2} .
\end{array}\right\}
$$

Take $z=(v, u) \in \mathscr{D}(K)$, so $v \in \mathscr{D}(T)$ and $u \in \mathscr{D}(S)$, and set $y=K z \in Y$, so $z=(v, u)=K^{-1} y$. From (4.2) and (4.3) we have

$$
\begin{aligned}
v^{(k)}(s) & =\left\langle K g_{k s}^{v}, K z\right\rangle \\
& =\left(R g_{k s}^{v 2}+T g_{k s}^{v 1}, R u+T v\right)+\left(S g_{k s}^{v 2}-g_{k s}^{v 1}, S u-v\right)
\end{aligned}
$$

and

$$
\begin{aligned}
u^{(j)}(s) & =\left\langle K g_{j s}^{u}, K z\right\rangle \\
& =\left(R g_{j s}^{u 2}+T g_{j s}^{u 1}, R u+T v\right)+\left(S g_{j s}^{u 2}-g_{j s}^{u 1}, S u-v\right),
\end{aligned}
$$

valid for all $z=(v, u)$ in $\mathscr{D}(K)=\mathscr{D}(T) \times \mathscr{D}(S)$. 
Let $G_{s}(t)=G(s, t)$ be the Green's function associated with $L$, and let

$$
G_{j s}(t)=\frac{\partial^{\prime}}{\partial s^{j}} G(s, t) .
$$

Take any $u \in \mathscr{D}(L)=\mathscr{D}(T S) \subseteq \mathscr{D}(S)$, and let $v=S u \in \mathscr{D}(T)$. From (4.5) we obtain

$$
u^{(j)}(s)=\left(R g_{j s}^{u 2}+T g_{j s}^{u 1}, L u\right)=\left(G_{j s}, L u\right) .
$$

Since $\mathscr{R}(L)=X$, we must have

$$
R g_{j s}^{u 2}+T g_{j s}^{u 1}=G_{j s}
$$

Setting $u=0$ in (4.7) we see that for each $v \in \mathscr{D}(T)$

$$
0=\left(R g_{j s}^{u 2}+T g_{j s}^{u 1}, T v\right)+\left(S g_{j s}^{u 2}-g_{j s}^{u 1},-v\right) .
$$

Hence, $R g_{j s}^{u 2}+T g_{j s}^{u 1} \in \mathscr{D}\left(T^{*}\right)$ with

$$
T^{*}\left(R g_{j s}^{u 2}+T g_{j s}^{u 1}\right)=S g_{j s}^{u 2}-g_{j s}^{u 1} \text {. }
$$

Combining (4.8) and (4.9), we have $G_{j s} \in \mathscr{D}\left(T^{*}\right)$ with

$$
S g_{j s}^{u 2}-g_{j s}^{u l}=T^{*} G_{j s}
$$

The error analysis of subsequent sections requires sufficient regularity of $\mathscr{G}_{j s}^{u}$ to invoke the error estimates of Section 2. Analysis of the smoothness of $\mathscr{G}_{j s}^{u}$ starts with that of $G_{j s}$. In particular, it is well known that for each $s \in[a, b]$ and for each $0 \leqslant j \leqslant m+n-1$

$$
G_{j s} \in H^{m+n-j-1}[a, b] \cap C^{\infty}[a, s] \cap C^{\infty}[s, b] .
$$

If we compare (4.4) to (4.8) and (4.10), then we see that

$$
\left\{\begin{array}{l}
\mathscr{G}_{j s}^{u 1}=G_{j s} \in H^{m+n-J-1}[a, b] \cap C^{\infty}[a, s] \cap C^{\infty}[s, b] \\
\mathscr{G}_{j s}^{u 2}=T^{*} G_{j s} \in H^{n-\jmath-1}[a, b] \cap C^{\infty}[a, s] \cap C^{\infty}[s, b] .
\end{array}\right\}
$$

Next, we apply our earlier methods to (4.8) and (4.10). In particular, apply $R S^{\dagger}$ to (4.10) to obtain

$$
R g_{j s}^{u 2}-R S^{\dagger} g_{j s}^{u 1}=R S^{\dagger} T^{*} G_{j s},
$$

and subtract this result from (4.8) to yield

$$
\left(T+R S^{\dagger}\right) g_{j s}^{u 1}=\tau g_{j s}^{u 1}=G_{j s}-R S^{\dagger} T^{*} G_{j s} .
$$

Clearly

$$
G_{j s}-R S^{\dagger} T^{*} G_{j s} \in H^{n-\jmath-1}[a, b] \cap C^{\infty}[a, s] \cap C^{\infty}[s, b] .
$$

We know that $g_{j s}^{u 1} \in \mathscr{D}(T) \subseteq H^{m}[a, b] \subseteq H^{1}[a, b]$, so $R S^{\dagger} g_{j s}^{u 1} \in H^{1}[a, b]$ and

$$
T g_{j s}^{\mu 1}=G_{j s}-R S^{\dagger} T^{*} G_{j s}-R S^{\dagger} g_{j s}^{\mu 1} \in H^{1}[a, b],
$$


which yields $g_{j s}^{u 1} \in H^{m+1}[a, b]$. Similarly, $g_{j s}^{u 1} \in H^{m+1}[a, b]$ gives $g_{j s}^{u 1} \in H^{2}[a, b]$, so $R S^{\dagger} g_{j s}^{u 1} \in H^{2}[a, b]$ and

$$
T g_{j s}^{u 1}=G_{j s}-R S^{\dagger} T^{*} G_{j s}-R S^{\dagger} g_{j s}^{u 1} \in H^{2}[a, b],
$$

which yields $g_{j s}^{\mu 1} \in H^{m+2}[a, b]$. Continuing this bootstrap argument, we conclude that

$$
\widetilde{g}_{j s}^{u 1} \in I^{m+n-j-1}[a, b] \cap C^{\approx}[a, s] \cap C^{\infty}[s, b] .
$$

Also, from (4.10), (4.12), and (4.13) we get

$$
g_{j s}^{u 2} \in H^{2 n-j-1}[a, b] \cap C^{\infty}[a, s] \cap C^{\infty}[s, b] .
$$

Summarizing these results, we obtain

TheOREM 4.1. For all $s \in[a, b]$ and $j=0,1, \ldots, n-1$ :

$$
g_{j s}^{u 1} \in H^{m+n-j-1}[a, b] \cap C^{\infty}[a, s] \cap C^{\infty}[s, b]
$$

and

$$
g_{j s}^{u 2} \in H^{2 n-J-1}[a, b] \cap C^{\infty}[a, s] \cap C^{\infty}[s, b] .
$$

These regularity results are critical to the analysis of convergence of SLS and Splitting Collocation procedures. Moreover, this same error analysis requires uniform boundedness of the derivatives $\left(g_{j s}^{u 1}\right)^{(p)}$ and $\left(g_{j s}^{u 2}\right)^{(p)}$ for $s \in[a, b]$ and for $p=0,1,2, \ldots$. To establish such uniform bounds, note that (4.12) implies

$$
\left(g_{j s}^{u 1}, g_{j s}^{u 2}\right)=K^{-1}\left(\mathscr{G}_{j s}^{u 1}, \mathscr{G}_{j s}^{u 2}\right)=K^{-1}\left(G_{j s}, T^{*} G_{j s}\right) .
$$

Hence, from the definition of $\lambda_{0 s}^{u}$ we obtain

$$
\begin{aligned}
g_{j s}^{u 2}(t) & =\lambda_{0 t}^{u}\left(G_{j s}, T^{*} G_{j s}\right)=\left.K^{-1}\left(G_{j s}, T^{*} G_{j s}\right)\right|_{t} \\
& =\left\langle G_{0 t}^{u},\left(G_{j s}, T^{*} G_{j s}\right)\right\rangle \\
& =\left\langle\left(G_{0 t}, T^{*} G_{0 t}\right),\left(G_{j s}, T^{*} G_{j s}\right)\right\rangle .
\end{aligned}
$$

In particular,

$$
g_{j s}^{\mu 2}(t)=\left(G_{0 t}, G_{j s}\right)+\left(T^{*} G_{0 t}, T^{*} G_{J s}\right)
$$

for all $t \in[a, b]$.

Using (4.15) together with bounds on the derivatives of $G_{j s}(t)$ and $T^{*} G_{j s}(t)$, we find that

$$
\sup _{s \in[a, b]}\left\|\left(g_{j s}^{u 2}\right)^{(p)}\right\|_{\infty}=\hat{\gamma}_{p}<\infty
$$

for $p=0,1,2, \ldots$. Also, from (4.10) we have

$$
g_{j s}^{u 1}(t)=S g_{j s}^{u 2}(t)-T^{*} G_{j s}(t)
$$


for all $t \in[a, b]$, and upon combining (4.16) and (4.17) we obtain

$$
\sup _{s \in[a, b]}\left\|\left(g_{j s}^{u !}\right)^{(p)}\right\|_{\infty}=\gamma_{p}<\infty
$$

for $p=0,1,2, \ldots$. Thus, we have

TheOREM 4.2. For $j=0,1, \ldots, n-1$ and for $p=0,1,2, \ldots$ :

$$
\sup _{s \in[a, b]}\left\|\left(g_{j s}^{u 1}\right)^{(p)}\right\|_{\infty}=\gamma_{p}<\infty
$$

and

$$
\sup _{s \in[a, b]}\left\|\left(g_{j s}^{\mu 2}\right)^{(p)}\right\|_{\infty}=\hat{\gamma}_{p}<\infty
$$

where $\gamma_{p}$ and $\hat{\gamma}_{p}$ are constants.

\section{Error analysis for splitting least squares}

Equations (4.1) and (4.2) yield the Green's matrix associated with the embedding operator $K$. In particular, if $K(v, u)=(\xi, \eta)$, then

$$
\begin{aligned}
& T v+R u=\xi, \\
& -v+S u=\eta,
\end{aligned}
$$

and

$$
\left[\begin{array}{l}
v^{(k)} \\
u^{(j)}
\end{array}\right]=\left[\begin{array}{ll}
K_{k}^{v 1} & K_{k}^{v 2} \\
K_{J}^{u 1} & K_{J}^{u 2}
\end{array}\right]\left[\frac{\xi}{\eta}\right] .
$$

The operators $K_{k}^{\nu 1}, K_{k}^{\nu 2}, K_{j}^{\mu 1}, K_{j}^{\mu 2}$ are integral operators with kernels $\mathscr{G}_{k}^{\nu 1}(s, \tau)$, $\mathscr{G}_{k}^{\nu 2}(s, \tau), \mathscr{G}_{J}^{u 1}(s, \tau)$, and $\mathscr{G}_{J}^{\mu 2}(s, \tau)$, respectively, where $a \leqslant s, \tau \leqslant b$. For example,

$$
K_{k}^{v 1} \xi(s)=\int_{a}^{b} \mathscr{G}_{k}^{v 1}(s, \tau) \xi(\tau) d \tau
$$

Alternately,

$$
\left[v^{(k)}(s), u^{(j)}(s)\right]=\left[\left\langle\mathscr{G}_{k s}^{v}, K z\right\rangle,\left\langle\mathscr{G}_{j s}^{u}, K z\right\rangle\right]
$$

for $z=(v, u) \in \mathscr{D}(K)$.

Let $\tilde{z}=(\tilde{v}, \tilde{u})$ be the SLS approximate to $z=(v, u)$ from $\mathscr{S}_{v} \times \mathscr{S}_{u}$ where $z$ solves $K z=(f, 0)$. For $0 \leqslant j \leqslant n-1$ and $0 \leqslant k \leqslant m-1$ equation (4.2) yields

$$
u^{(j)}(s)-\tilde{u}^{(j)}(s)=\left\langle\mathscr{G}_{j s}^{u}, K z-K \tilde{z}\right\rangle
$$

and

$$
v^{(k)}(s)-\tilde{v}^{(k)}(s)=\left\langle\mathscr{G}_{k s}^{v}, K z-K \tilde{z}\right\rangle
$$


where $u$ is the solution to the unsplit operator equation $L u=f$ and $v=S u$. We want to estimate (5.2) in both the $L^{\infty}[a, b]$ and $L^{2}[a, b]$ norms. By (4.3) $\mathscr{G}_{j s}^{u}=K g_{j s}^{u}$, so

$$
u^{(J)}(s)-\tilde{u}^{(j)}(s)=\left\langle K g_{j s}^{u}, K z-K \tilde{z}\right\rangle
$$

Moreover, by (2.5)

$$
\langle K \psi, K z-K \tilde{z}\rangle-0
$$

for all $\psi \in \mathscr{S}_{v} \times \mathscr{S}_{u}$, and hence,

$$
u^{(j)}(s)-\tilde{u}^{(j)}(s)=\left\langle K g_{j s}^{u}-K \tilde{g}_{j s}^{u}, K z-K \tilde{z}\right\rangle
$$

where $\tilde{g}_{j s}^{u} \in \mathscr{S}=\mathscr{S}_{v} \times \mathscr{S}_{u}$ satisfies

$$
\left|K g_{j s}^{u}-K \tilde{g}_{j s}^{u}\right|=\min _{\psi \in \mathscr{S}}\left|K g_{j s}^{u}-K \psi\right|
$$

From the Schwarz inequality applied to (5.4), we have

$$
\left|u^{(j)}(s)-\tilde{u}^{(j)}(s)\right| \leqslant\left|K g_{j s}^{u}-K \tilde{g}_{j s}^{u}\right| \cdot|K z-K \tilde{z}|,
$$

with (5.5) valid for all $s \in[a, b]$ and $j=0,1, \ldots, n-1$.

Now let $\mathscr{S}_{v}=\operatorname{Sp}(2 m-1, \Delta, m-1) \cap \mathscr{D}(T)$ and let

$$
\mathscr{S}_{u}=\operatorname{Sp}(2 n-1, \Delta, n-1) \cap \mathscr{D}(S) .
$$

Letting $\bar{z}=(\bar{v}, \bar{u})$ be the Hermite interpolate to $z=(v, u)$ from $\mathscr{S}_{v} \times \mathscr{S}_{u}$, we have

$$
\begin{aligned}
|K z-K \tilde{z}| & \leqslant|K z-K \bar{z}| \\
& =\left\{\|T(v-\bar{v})+R(u-\bar{u})\|^{2}+\|S(u-\bar{u})+(\bar{v}-v)\|^{2}\right\}^{1 / 2} \\
& \leqslant \beta\left\{\|u-\bar{u}\|_{n}+\|v-\bar{v}\|_{m}\right\}
\end{aligned}
$$

where $\beta=\left\{(\|T\|+\|R\|)^{2}+(1+\|S\|)^{2}\right\}^{1 / 2}$.

Now assume $f \in H_{\Delta}^{0, k}[a, b]$ where $0 \leqslant k \leqslant m$. The theory of ordinary differential operators implies

$$
u \in H_{\Delta}^{m+n, k}[a, b]
$$

and

$$
v \in H_{\Delta}^{m, k}[a, b] .
$$

Throughout the sequel let $\gamma$ denote a generic constant independent of $u, v$, and $h$. The error estimates of Theorem 2.2 imply

$$
\left\|u^{(j)}-\bar{u}^{(J)}\right\| \leqslant \gamma\left\|u^{(m+n+\sigma)}\right\| h^{m+n+\sigma-J}
$$

for $0 \leqslant j \leqslant m+n+\sigma$ where $\sigma=\min \{k, n-m\}$. In particular,

$$
\|u-\bar{u}\|_{n} \leqslant \gamma\left\|u^{(m+n+\sigma)}\right\| h^{m+\sigma}
$$

with

$$
\sigma=\min \{k, n-m\}
$$


Similarly, Theorem 2.2 implies

$$
\left\|v^{(\jmath)}-\bar{v}^{(\jmath)}\right\| \leqslant \gamma\left\|v^{(m+\tau)}\right\| h^{m+\tau-\jmath}
$$

for $0 \leqslant j \leqslant m+\tau$ where $\tau=\min \{m, k\}=k$, and hence,

$$
\|v-\bar{v}\|_{m} \leqslant \gamma\left\|v^{(m+k)}\right\| h^{k} .
$$

Combining (5.6), (5.9), and (5.12), we have

LEMMA 5.1. If $f \in H_{\Delta}^{0, k}[a, b]$, then

$$
|K z-K \tilde{z}| \leqslant \gamma \beta \mu_{u} h^{k},
$$

where $\mu_{u} \leqslant \gamma \max \left\{h^{p} A, B\right\}, A=\left\|u^{(m+n+\min \{k, n-m\})}\right\|, p=m+\min \{k, n-m\}$ $-k$, and $B=\left\|u^{(m+n+k)}\right\|$.

We next estimate $\left|K g_{j s}^{u}-K \tilde{g}_{j s}^{u}\right|$ where $\tilde{g}_{j s}^{u} \in \mathscr{S}_{v} \times \mathscr{S}_{u}$. Due to the regularity and uniform boundedness properties of $g_{j s}^{u}$ given in Theorems 4.1 and 4.2, it turns out that these estimates are order of best approximation when $s \in \Delta$. In particular, for $s \in \Delta$ Theorem 4.1 gives

$$
g_{j s}^{u 1} \in H_{\Delta}^{m, k}[a, b]
$$

and

$$
g_{j s}^{u 2} \in H_{\Delta}^{n, \prime}[a, b]
$$

for each $j=0,1, \ldots, n-1$ and for all $k$ and $l=0,1,2, \ldots$ Let $\bar{g}_{j s}^{u}=\left(\bar{g}_{j s}^{u 1}, \bar{g}_{j s}^{u 2}\right)$ be the Hermite interpolate to $g_{j s}^{u}$ from $\mathscr{S}_{v} \times \mathscr{S}_{u}$. We know that

$$
\left|K g_{j s}^{u}-K \tilde{g}_{j s}^{u}\right| \leqslant\left|K g_{j s}^{u}-K \bar{g}_{j s}^{u}\right| \text {. }
$$

Letting $k=m$ and $l=n$ with $s \in \Delta$, Theorem 2.2 combined with (5.13) and (5.14) yields

$$
\left\|\left(g_{j s}^{u 1}\right)^{(p)}-\left(\bar{g}_{j s}^{u 1}\right)^{(p)}\right\| \leqslant \gamma\left\|\left(g_{j s}^{u 1}\right)^{(2 m)}\right\| h^{2 m-p}
$$

and

$$
\left\|\left(g_{j s}^{u 2}\right)^{(q)}-\left(\bar{g}_{j s}^{u 2}\right)^{(q)}\right\| \leqslant \gamma\left\|\left(g_{j s}^{u 2}\right)^{(2 n)}\right\| h^{2 n-q}
$$

for $j=0,1, \ldots, n-1$, for $p=0,1, \ldots, 2 m$, and for $q=0,1, \ldots, 2 n$. Invoking Theorem 4.2, we have

$$
\left\|g_{j s}^{u 1}-\bar{g}_{j s}^{u 1}\right\|_{m} \leqslant \gamma \cdot \gamma_{2 m} h^{m}
$$

and

$$
\left\|g_{j s}^{u 2}-\bar{g}_{j s}^{u 2}\right\|_{n} \leqslant \gamma \cdot \hat{\gamma}_{2 n} h^{n}
$$


for $j=0,1, \ldots, n-1$. Combining (5.15), (5.16), and (5.17) we have (recall $m \leqslant n)$

LEMMA 5.2. For each $s \in \Delta$ and $j=0,1, \ldots, n-1$,

$$
\left|K g_{j s}^{u}-K \tilde{g}_{j s}^{u}\right| \leqslant \gamma \cdot \beta \cdot h^{m} .
$$

Combining Lemmas 5.1 and 5.2 and (5.5), we have

THEOREM 5.3. (Superconvergence at spline knots). If $u=L^{-1} f \in H_{\Delta}^{m+n, k}[a, b]$ with $0 \leqslant k \leqslant m$, then for each $s_{1} \in \Delta, i=0,1, \ldots, N$, and each $j=0,1, \ldots, n-1$

$$
\left|u^{(J)}\left(s_{\imath}\right)-\tilde{u}^{(j)}\left(s_{i}\right)\right| \leqslant \gamma \beta^{2} \mu_{u} h^{m+k} .
$$

Combining Theorems 5.3, 2.2, and 2.3 with Markov's inequality (see [25, 15, 16]) yields the following global error estimates for SLS.

THEOREM 5.4. If $u=L^{-1} f \in H_{\Delta}^{m+n, k}[a, b]$ with $0 \leqslant k \leqslant m$, then

$$
\left\|u^{(j)}-\tilde{u}^{(j)}\right\| \leqslant \gamma \mu_{u} h^{m+k-j}
$$

for $j=0,1, \ldots, m+k$. If $u=L^{-1} f \in C_{\Delta}^{m+n, k}[a, b]$ with $0 \leqslant k \leqslant m$, then

$$
\left\|u^{(j)}-\tilde{u}^{(\jmath)}\right\|_{\infty} \leqslant \gamma \mu_{u} h^{m+k-\jmath}
$$

for $j=0,1, \ldots, m+k$.

\section{Splitting collocation}

We now consider the approximate solution of $L u=f$ via collocation schemes applied to the split equations (1.3) and (1.4). To simplify the analysis of this section, we assume the coefficients of $L$ are in $C^{\infty}[a, b]$, although much weaker conditions will also suffice. To describe the method, we start with the partition $\Delta$ : $a=t_{0}<t_{1}<\cdots<t_{N}=b$ of $[a, b]$, where $t_{1}=s_{i}$ in our previous notation. On each subinterval $I_{t}=\left[t_{t-1}, t_{i}\right]$ of $\Delta$, let

$$
t_{i 1}^{k}<t_{t 2}^{k}<\cdots<t_{i k}^{k}
$$

be the Gauss points of $I_{t}$ and let $\left\{w_{t 1}^{k}, w_{i 2}^{k}, \ldots, w_{i k}^{k}\right\}$ be the weights associated with $k$ th order Gaussian quadrature on $I_{i}$. Then

$$
\int_{t_{i-1}}^{t_{i}} q(t) d t=\sum_{j=1}^{k} w_{i j}^{k} q\left(t_{i j}^{k}\right)
$$


whenever $q$ is a polynomial of degree $2 k-1$. Well-known bounds on the error in such Gaussian quadrature are given by

$$
\left|\int_{t_{i-1}}^{t_{i}} q(t) d t-\sum_{j=1}^{k} w_{i j}^{k} q\left(t_{i j}^{k}\right)\right| \leqslant \gamma h^{2 k} \int_{t_{i-1}}^{t_{i}}\left|D^{2 k}[q(t)]\right| d t,
$$

where $h=\max \left\{t_{i}-t_{i-1}: i=1,2, \ldots, N\right\}, D^{2 k}=d^{2 k} / d t^{2 k}$, and $q \in H^{2 k}\left[t_{1-1}, t_{t}\right]$ for each $i=1,2, \ldots, N$.

To solve $L u=f$ by splitting collocation, we seek $(\hat{v}, \hat{u}) \in \mathscr{S}_{v} \times \mathscr{S}_{u}$ solving the linked collocation system

$$
\begin{gathered}
(T \hat{v}+R \hat{u})\left(t_{i j}^{m}\right)=f\left(t_{t j}^{m}\right), \\
(\hat{v}-S \hat{u})\left(t_{t l}^{n}\right)=0,
\end{gathered}
$$

where $1 \leqslant i \leqslant N, 1 \leqslant j \leqslant m$, and $1 \leqslant l \leqslant n$. In order that (6.2) and (6.3) be well defined, we assume that $f \in C_{\Delta}^{0, k}[a, b]$ for some integer $k \geqslant 0$.

We shall prove that the linked system (6.2)-(6.3) is uniquely solvable by showing that splitting collocation is equivalent to splitting discrete least squares. To this end we introduce the pseudo-inner product and norm on $C_{\Delta}^{0,0}[a, b] \times$ $C_{\Delta}^{0,0}[a, b]=\mathscr{X}^{0}$

$$
\langle z, Z\rangle_{*}=\langle v, V\rangle_{m}+\langle u, U\rangle_{n}, \quad|z|_{*}=\sqrt{\langle z, z\rangle_{*}},
$$

where $z=(v, u)$ and $Z=(V, U)$, and where $\langle,\rangle_{m},||_{m},\langle,\rangle_{n}$, and ||$_{n}$ are the pseudo-inner products and norms

$$
\langle v, V\rangle_{m}=\sum_{i=1}^{N} \sum_{j=1}^{m} w_{i j}^{m} v\left(t_{i j}^{m}\right) V\left(t_{i j}^{m}\right), \quad|v|_{m}=\sqrt{\langle v, v\rangle_{m}},
$$

$v, V \in C_{\Delta}^{0,0}[a, b]$, and

$$
\langle u, U\rangle_{n}=\sum_{i=1}^{N} \sum_{j=1}^{n} w_{t j}^{n} u\left(t_{t j}^{n}\right) U\left(t_{i j}^{n}\right), \quad|u|_{n}=\sqrt{\langle u, u\rangle_{n}},
$$

$u, U \in C_{\Delta}^{0,0}[a, b]$. The method of discrete splitting least squares seeks $\hat{z} \in \mathscr{S}_{v} \times \mathscr{S}_{u}$ $=\mathscr{S}_{\text {solving }}$

$$
|K \hat{z}-y|_{*}=\min _{w \in \mathscr{S}^{\infty}}|K w-y|_{*}
$$

where $y=(f, 0)$.

THEOREM 6.1. If $m=n$ or $p<n$, then equation (6.4) is uniquely solvable for all $h$ sufficiently small. 
Proof. Existence of $\hat{z}$ solving (6.4) is guaranteed by simple finite-dimensional arguments. To show uniqueness, it suffices to prove $|K \hat{z}|_{*}=0$ implies $\hat{z}=(0,0)$. Letting $\hat{z}=(\hat{v}, \hat{u}) \in \mathscr{S}_{v} \times \mathscr{S}_{u}$, we have

$$
\left.|| K \hat{z}\right|^{2}-|K \hat{z}|_{*}^{2}|=|\|T \hat{v}+R \hat{u}\|^{2}-|T \hat{v}+R \hat{u}|_{m}^{2}+\|\hat{v}-S \hat{u}\|^{2}-|\hat{v}-S \hat{u}|_{n}^{2} \mid .
$$

The right-hand side of $(6.5)$ is the sum of the terms

$$
\begin{gathered}
\|T \hat{v}\|^{2}-|T \hat{v}|_{m}^{2}, \\
\|R \hat{u}\|^{2}-|R \hat{u}|_{m}^{2}, \\
2(T \hat{v}, R \hat{u})-2\langle T \hat{v}, R \hat{u}\rangle_{m},
\end{gathered}
$$

and

$$
\|S \hat{u}-\hat{v}\|^{2}-|S \hat{u}-\hat{v}|_{n}^{2} .
$$

The most difficult term to estimate is (6.7). With this in mind we analyze this case in some detail and leave the other terms to the reader. Let $\gamma, \gamma_{1}, \ldots$ be generic constants independent of $u, v$, and $h$. Applying the error estimate (6.1) to (6.7), we find

$$
\begin{aligned}
\left.\left|\|R \hat{u}\|^{2}-\right| R \hat{u}\right|_{m} ^{2} \mid & \leqslant \gamma_{1} h^{2 m} \sum_{i=1}^{N} \int_{t_{t-1}}^{t_{i}}\left|D^{2 m}[R \hat{u}]^{2}\right| d t \\
& \leqslant \gamma_{2} h^{2 m} \sum_{l=0}^{2 m}\left(\begin{array}{c}
2 m \\
l
\end{array}\right)\|\hat{u}\|_{0,2 m+p-l}\|\hat{u}\|_{0, p+l} .
\end{aligned}
$$

Noting that $D^{k} \hat{u}=0$ for all $k \geqslant 2 n$ and invoking Schmidt's inequality $[2,15,16]$, for $m=n$ we obtain

$$
\begin{aligned}
\left.\left|\|R \hat{u}\|^{2}-\right| R \hat{u}\right|_{m} ^{2} \mid & \leqslant \gamma_{3} h^{2 m}\|\hat{u}\|_{n, n-1}^{2} \\
& \leqslant \gamma_{3} h^{2 m}\left[\gamma_{4} h^{1-n}\|\hat{u}\|_{n}^{2}\right] \\
& =\gamma_{5} h^{2}\|\hat{u}\|_{n}^{2} \\
& \leqslant \gamma_{5} h\|\hat{z}\|^{2} .
\end{aligned}
$$

For $m<n$ and $p<n$ we must carefully estimate the quantities

$$
h^{2 m}\|\hat{u}\|_{0,2 m+p-l}\|\hat{u}\|_{0, p+l}
$$

by considering the cases: (i) $2 m+p-l \leqslant n$ with $p+l \leqslant n$, (ii) $2 m+p-l \leqslant n$ with $p+l>n$, (iii) $2 m+p-l>n$ with $p+l \leqslant n$, and (iv) $2 m+p-l>n$ with $p+l>n$. We carry out case (ii), omitting the other cases which are similar. Assume $2 m+p-l \leqslant n$ with $p+l>n$. Clearly

$$
\|\hat{u}\|_{0,2 m+p-l} \leqslant\|\hat{u}\|_{n} .
$$


If $n<p+l \leqslant 2 n-1$, then

$$
\|\hat{u}\|_{0, p+l}=\|\hat{u}\|_{n, p+l-n} \leqslant \gamma_{6} h^{-(p+l-n)}\|\hat{u}\|_{n},
$$

while if $2 n-1<p+l$, then

$$
\|\hat{u}\|_{0, p+l}=\|\hat{u}\|_{n, n-1} \leqslant \gamma_{7} h^{-(n-1)}\|\hat{u}\|_{n} \leqslant \gamma_{7} h^{-(p+l-n)}\|\hat{u}\|_{n} .
$$

Thus,

$$
\begin{aligned}
h^{2 m}\|\hat{u}\|_{0,2 m+p-l}\|\hat{u}\|_{0, p+l} & \leqslant h^{2 m}\|\hat{u}\|_{n} \gamma_{8} h^{-(p+l-n)}\|\hat{u}\|_{n} \\
& =\gamma_{8} h^{(2 m-l)+(n-p)}\|\hat{u}\|_{n}^{2} \\
& \leqslant \gamma_{8} h\|\hat{u}\|_{n}^{2}
\end{aligned}
$$

or

$$
h^{2 m}\|\hat{u}\|_{0,2 m+p-l}\|\hat{u}\|_{0, p+l} \leqslant \gamma h\|\hat{z}\|^{2}
$$

for $0 \leqslant l \leqslant 2 m$. Similar arguments establish (6.11) in the remaining cases.

Combining (6.10) with (6.11) gives

$$
\left.\left|\|R \hat{u}\|^{2}-\right| R u\right|_{m} ^{2}|\leqslant \gamma h|\|\hat{z}\|^{2} .
$$

The same argument yields

$$
\begin{gathered}
\left.\left|\|T \hat{v}\|^{2}-\right| T \hat{v}\right|_{m} ^{2} \mid \leqslant \gamma h\|\hat{z}\| \|^{2}, \\
\left|(T \hat{v}, R \hat{u})-\langle T \hat{v}, R \hat{u}\rangle_{m}\right| \leqslant \gamma h\|\hat{z}\|^{2}, \\
\left|\|S \hat{u}-\hat{v}\|^{2}-\right| S \hat{u}-\left.\hat{v}\right|_{n} ^{2}|\leqslant \gamma h\|\hat{z}\||^{2} .
\end{gathered}
$$

Combining (6.12)-(6.15) with (6.5), we see that

$$
\left.|| K \hat{z}\right|^{2}-|K \hat{z}|_{*}^{2} \mid \leqslant \gamma h\|\hat{z}\| \|^{2} \text {. }
$$

But (6.16) implies

$$
\begin{aligned}
|K \hat{z}|_{*}^{2} & \geqslant|K \hat{z}|^{2}-\gamma\|\hat{z}\|^{2} \\
& \geqslant\left.\gamma_{0}\|\hat{z}\|\right|^{2}-\gamma h\|\hat{z}\|^{2} \\
& =\left[\gamma_{0}-\gamma h\right]\|\hat{z}\|^{2}
\end{aligned}
$$

where $\gamma_{0}=1 /|| K^{-1} \|^{2}$. It follows that if $|K \hat{z}|_{*}=0$, then

$$
\|\hat{z}\|=\|\hat{v}\|_{m}+\|\hat{u}\|_{n}=0
$$

for $h$ sufficiently small. Thus, $\hat{z}=(0,0)$. Q.E.D.

COROllaRy 6.2. For $m=n$ or $p<n$ and for all $\hat{z} \in \mathscr{S}_{v} \times \mathscr{S}_{u}$,

$$
|K \hat{z}|_{*}^{2} \geqslant\left(\gamma_{0}-\gamma h\right)\|\mid \hat{z}\|^{2} \text {. }
$$


Proof. This inequality was established in the proof of Theorem 6.1. Q.E.D.

To connect discrete splitting least squares to splitting collocation, note that if $\tilde{z}=(\tilde{v}, \tilde{u}) \in \mathscr{S}$ solves (6.2) and (6.3), then

$$
0=|K \tilde{z}-y|_{*}=\min _{w \in \mathscr{S}}|K w-y|_{*}=|K \hat{z}-y|_{*} .
$$

in particuiar, given the existence of $\tilde{z}$, for all $h$ sufficiently small $\tilde{z}=\hat{z}$ and is unique. Moreover, whenever the homogeneous collocation equations (i.e. $f=0$ in (6.2)) are uniquely solvable, the nonhomogeneous linked collocation equations are uniquely solvable and the linked collocation matrix is nonsingular. Thus, we have proved

THEOREM 6.3. For $m=n$ or $p<n$ the linked collocation equations (6.2) and (6.3) are uniquely solvable for all $h$ sufficiently small. Moreover, in this context the splitting linked collocation and splitting discrete least squares approximates to $\mathbf{L u}=f$ are identical.

Just as in split least squares, the split collocation error $u^{(\jmath)}-\hat{u}^{(\jmath)}$ experiences superconvergence at spline knodal points. In proving these results we will use

LemMa 6.4. Let $u=L^{-1} f \in C_{\Delta}^{m+n, m+n}[a, b]$, let $\hat{z}=(\hat{v}, \hat{u})$ be the unique split collocation approximate to $z=(v, u)$ with $v=S u$, and let $\bar{z}=(\bar{v}, \bar{u})$ be the Hermite interpolate to $z=(v, u)$. Then

$$
\|\hat{z}-\bar{z}\| \leqslant \gamma h^{m}\|u\|_{0,2 m+2 n},
$$

where $\gamma$ is a constant independent of $u, v$, and $h$.

Proof. Corollary 6.2 and (6.2)-(6.3) give

$$
\|\hat{z}-\bar{z}\|\left|\leqslant \gamma_{1}\right| K \hat{z}-\left.K \bar{z}\right|_{*}=\gamma_{1}|K z-K \bar{z}|_{*} .
$$

For the right-hand term we have

$$
\begin{aligned}
|K z-K \bar{z}|_{*}^{2}= & |T v-T \bar{v}+R u-R \bar{u}|_{m}^{2}+|v-\bar{v}+S \bar{u}-S u|_{n}^{2} \\
= & \sum_{i=1}^{n} \sum_{j=1}^{m} w_{i j}^{m}\left[(T v-T \bar{v})\left(t_{i j}^{m}\right)+(R u-R \bar{u})\left(t_{i j}^{m}\right)\right]^{2} \\
& +\sum_{i=1}^{N} \sum_{j=1}^{n} w_{i j}^{n}\left[(v-\bar{v})\left(t_{i j}^{n}\right)+(S \bar{u}-S u)\left(t_{i j}^{n}\right)\right]^{2} \\
\leqslant & {\left[\|T v-T \bar{v}\|_{\infty}+\|R u-R \bar{u}\|_{\infty}\right]^{2}(b-a) } \\
& +\left[\|v-\bar{v}\|_{\infty}+\|S u-S \bar{u}\|_{\infty}\right]^{2}(b-a),
\end{aligned}
$$


where the quadrature weights sum to the interval length. Since $u \in$ $C_{\Delta}^{m+n, m+n}[a, b]$, the above inequality coupled with Theorem 2.3 yields

$$
|K z-K \bar{z}|_{*} \leqslant \gamma_{2} h^{m}|| u \|_{0,2 m+2 n} .
$$

The result follows from (6.17) and (6.18). Q.E.D.

THEOREM 6.5. (Superconvergence at spline knots). Let $u=L^{-1} f \in C_{\Delta}^{m+n, 2 n}[a, b]$ with $m=n$ or $p<n$. Then for each $s \in \Delta$ and $j=0,1, \ldots, n-1$ :

$$
\left|u^{(j)}(s)-\hat{u}^{(j)}(s)\right| \leqslant \begin{cases}\gamma h^{2 m}\|u\|_{0, m+3 n} & \text { for } m=n, m=n-1, \\ \gamma h^{\min \{2 m, m+n-p\}}\|u\|_{0, m+3 n} & \text { for } m+p \leqslant n,\end{cases}
$$

Proof. For $s \in \Delta$ and $j=0,1, \ldots, n-1$ we note that

$$
\left\langle K g_{j s}^{u}, K z-K \hat{z}\right\rangle_{*}=0
$$

by (6.2) and (6.3). Hence,

$$
u^{(j)}(s)-\hat{u}^{(j)}(s)=\left\langle K g_{j s}^{u}, K z-K \hat{z}\right\rangle=E_{j}(s),
$$

where

$$
E_{j}(s)=E_{1 j}(s)+E_{2 j}(s)
$$

is the quadrature error. Specifically,

$$
\begin{aligned}
E_{1}(s)= & \left(T g_{j s}^{u 1}+R g_{j s}^{u 2}, T v-T \hat{v}+R u-R \hat{u}\right) \\
& -\left\langle T g_{j s}^{u 1}+R g_{j s}^{u 2}, T v-T \hat{v}+R u-R \hat{u}\right\rangle_{m}
\end{aligned}
$$

and

$$
\begin{aligned}
E_{2 j}(s)= & \left(g_{j s}^{u 1}-S g_{j s}^{u 2}, v-\hat{v}+S \hat{u}-S u\right) \\
& -\left\langle g_{j s}^{u 1}-S g_{j s}^{u 2}, v-\hat{v}+S \hat{u}-S u\right\rangle_{n} .
\end{aligned}
$$

We must bound $E_{1 j}(s)$ and $E_{2 j}(s)$ for $s \in \Delta$. Invoking Theorems 4.1 and 4.2 together with (6.1), we have

$$
\begin{aligned}
\left|E_{1 j}(s)\right| & \leqslant \gamma_{1} h^{2 m} \sum_{l=0}^{2 m}\left(\begin{array}{c}
2 m \\
l
\end{array}\right) \int_{a}^{b}\left|D^{\prime}(T v-T \hat{v}+R u-R \hat{u})\right| d t \\
& \leqslant \gamma_{2} h^{2 m}\left\{\|T v-T \hat{v}\|_{0,2 m}+\|R u-R \hat{v}\|_{0,2 m}\right\} \\
& \leqslant \gamma_{3} h^{2 m}\left\{\|v-\hat{v}\|_{0,3 m}+\|u-\hat{u}\|_{0,2 m+p}\right\} .
\end{aligned}
$$

Similarly,

$$
\left|E_{2 j}(s)\right| \leqslant \gamma_{4} h^{2 n}\left\{\|v-\hat{v}\|_{0,2 n}+\|u-\hat{u}\|_{0,3 n}\right\} .
$$


We first bound the terms $\|v-\hat{v}\|_{0, k}$ where $k=3 m$ or $k=2 n$. Take any integer $k \geqslant 2 m-1$. Let $\bar{v} \in \mathscr{S}_{v}$ be the Hermite interpolate to $v$. Observing that $S u=v$ and invoking Schmidt's inequality (see $[15,16]$ ) together with Lemma 6.4 , we have

$$
\begin{aligned}
\|v-\hat{v}\|_{0, k} & \leqslant\|v-\bar{v}\|_{0, k}+\|\bar{v}-\hat{v}\|_{0, k} \\
& \leqslant \gamma_{5}\|v\|_{0, k}+\|\bar{v}-\hat{v}\|_{0,2 m-1} \\
& \leqslant \gamma_{5}\|v\|_{0, k}+\gamma_{6} h^{1-m}\|\bar{v}-\hat{v}\|_{m} \\
& \leqslant \gamma_{7}\|u\|_{0, k+n}+\gamma_{6} h^{1-m}\|\bar{z}-\hat{z}\| \\
& \leqslant \gamma_{7}\|u\|_{0, k+n}+\gamma_{8} h\|u\|_{0,2 m+2 n} .
\end{aligned}
$$

For $k=3 m$ and $k=2 n$ it follows that

$$
\|v-\hat{v}\|_{0,3 m} \leqslant \gamma_{9}\|u\|_{0,2 m+2 n},
$$

while

$$
\|v-\hat{v}\|_{0,2 n} \leqslant \gamma_{10}\|u\|_{0, m+3 n} .
$$

Combining (6.22), (6.23), (6.24), and (6.25), we have

$$
\left|E_{1}(s)\right| \leqslant h^{2 m}\left[\gamma_{11} \mid\|u\|_{0,2 m+2 n}+\gamma_{3}\|u-\hat{u}\|_{0,2 m+p}\right]
$$

and

$$
\left|E_{2 j}(s)\right| \leqslant h^{2 n}\left[\gamma_{12}\|u\|_{0, m+3 n}+\gamma_{4}\|u-\hat{u}\|_{0,3 n}\right] .
$$

Tight bounds on the terms $\|u-\hat{u}\|_{0, k}$ for $k=2 m+p$ or $k=3 n$ are more difficult to obtain, and the estimates of this theorem divide into three cases: (i) $m=n$ or $m=n-1$, (ii) $m+p \leqslant n$, and (iii) $m+p>n$. The special case when $\mathscr{R}(S)=L^{2}[a, b]$ is covered in the next theorem. Before going into the cases, note that if $\bar{u} \in \mathscr{S}_{u}$ is the Hermite interpolate to $u$, then invoking Schmidt's inequality and Lemma 6.4, we have

$$
\begin{aligned}
\|u-\hat{u}\|_{0,3 n} & \leqslant\|u-\bar{u}\|_{0,3 n}+\|\bar{u}-\hat{u}\|_{0,3 n} \\
& \leqslant \gamma_{13}\|u\|_{0,3 n}+\|\bar{u}-\hat{u}\|_{0,2 n-1} \\
& \leqslant \gamma_{13}\|u\|_{0,3 n}+\gamma_{14} h^{1-n}\|\bar{u}-\hat{u}\|_{n} \\
& \leqslant \gamma_{13}\|u\|_{0,3 n}+\gamma_{14} h^{1-n}\|\bar{z}-\hat{z}\| \\
& \leqslant \gamma_{15} h^{m-n+1}\|u\|_{0, m+3 n} .
\end{aligned}
$$

Thus,

$$
h^{2 n}\|u-\hat{u}\|_{0,3 n} \leqslant \gamma_{15} h^{m+n+1}\|u\|_{0, m+3 n},
$$

and combining (6.27) with (6.29), we have proved

$$
\left|E_{2,}(s)\right| \leqslant \gamma h^{m+n}\|u\|_{0, m+3 n} .
$$


To estimate the term $\left|E_{1 j}(s)\right|$, we must bound the term $h^{2 m}\|u-\hat{u}\|_{0,2 m+p}$. Starting with the case $m=n$ or $m=n-1$ and invoking (6.28), we have

$$
\begin{aligned}
\|u-\hat{u}\|_{0,2 m+p} & \leqslant\|u-\hat{u}\|_{0,3 n} \leqslant \gamma_{15} h^{m-n+1}\|u\|_{0, m+3 n} \\
& \leqslant \gamma_{15}\|u\|_{0, m+3 n}
\end{aligned}
$$

for $h \leqslant 1$. Thus,

$$
h^{2 m}\|u-\hat{u}\|_{0,2 m+p} \leqslant \gamma h^{2 m}\|u\|_{0, m+3 n}, \quad m=n \text { or } m=n-1 .
$$

Next, observe that

$$
\begin{aligned}
\|u-\hat{u}\|_{0,2 m+p} & \leqslant\|u-\bar{u}\|_{0,2 m+p}+\|\bar{u}-\hat{u}\|_{0,2 m+p} \\
& \leqslant \gamma_{16}\|u\|_{0,2 m+p}+\|\bar{u}-\hat{u}\|_{0,2 m+p} .
\end{aligned}
$$

Invoking Schmidt's inequality, we have

$$
\begin{aligned}
\|\bar{u}-\hat{u}\|_{0,2 m+p} & =\|\bar{u}-\hat{u}\|_{0, n+m+(m+p-n)} \\
& \leqslant \gamma_{17} h^{-m}\|\bar{u}-\hat{u}\|_{0, n+(m+p-n)} .
\end{aligned}
$$

If $m+p-n \leqslant 0$, then from Lemma 6.4

$$
\begin{aligned}
\|\bar{u}-\hat{u}\|_{0, n+(m+p-n)} & \leqslant\|\bar{u}-\hat{u}\|_{n} \leqslant\|\bar{z}-\hat{z}\| \\
& \leqslant \gamma_{18} h^{m}\|u\|_{0,2 m+2 n} .
\end{aligned}
$$

Combining (6.32), (6.33), and (6.34), we have

$$
h^{2 m}\|u-\hat{u}\|_{0,2 m+p} \leqslant \gamma h^{2 m}\|u\|_{0,2 m+2 n} \text { for } m+p \leqslant n .
$$

On the other hand, if $m+p>n$, then

$$
\begin{aligned}
h^{-m}\|\bar{u}-\hat{u}\|_{0, n+(m+p-n)} & \leqslant \gamma_{19} h^{n-p-2 m}\|\bar{u}-\hat{u}\|_{n} \\
& \leqslant \gamma_{19} h^{n-p-2 m}\|\bar{z}-\hat{z}\| \\
& \leqslant \gamma_{20} h^{n-p-m}\|u\|_{0,2 m+2 n} .
\end{aligned}
$$

Combining (6.32), (6.33), and (6.35), we find that

$$
h^{2 m}\|u-\hat{u}\|_{0,2 m+p} \leqslant \gamma h^{\min \{2 m, m+n-p)}\|u\|_{0,2 m+2 n}
$$

for $m+p>n$. Equations (6.26), (6.31), (6.35), and (6.37) yield

$$
\left|E_{1}(s)\right| \leqslant \begin{cases}\gamma h^{2 m}\|u\|_{0, m+3 n} & \text { for } m=n, m=n-1, \\ \gamma h^{\min \{2 m, m+n-p)}\|u\|_{0, m+3 n} & \text { for } m+p \leqslant n\end{cases}
$$

Inequalities (6.30), (6.38), and equation (6.19) prove the theorem. Q.E.D.

In the event $S$ is invertible, we obtain another condition under which superconvergence is order $h^{2 m}$. Specifically, we have

THEOREM 6.6. Let $u=L^{-1} f \in C_{\Delta}^{m+n, 2 n}[a, b]$ with $m=n$ or $p<n$. Assume $\mathscr{R}(S)=L^{2}[a, b]$, so that $S$ is invertible. Then for each $s \in \Delta$ and $j=0,1, \ldots, n-1$ :

$$
\left|u^{(j)}(s)-\hat{u}^{(j)}(s)\right| \leqslant \gamma h^{2 m}\|u\|_{0, m+3 n} .
$$


Proof. Since $\mathscr{R}(S)=L^{2}[a, b], \hat{v} \in \mathscr{R}(S)$. Let $S w=\hat{v}$, so that

$$
S w=\hat{v} \text { and } S u=v .
$$

Since $\hat{v} \in C_{\Delta}^{m, k}[a, b]$ for all $k \geqslant 0$, it follows that

$$
w \in C_{\Delta}^{m+n, k}[a, b] \text { for all } k \geqslant 0,
$$

while

$$
u \in C_{\Delta}^{m+n, 2 n}[a, b]
$$

by assumption. Moreover, for $i=1,2, \ldots, N$ and $j=1,2, \ldots, n$

$$
S \hat{u}\left(t_{i j}^{n}\right)=\hat{v}\left(t_{i j}^{n}\right)=S w\left(t_{i j}^{n}\right) .
$$

Thus, $\hat{u} \in \mathscr{S}_{u}$ is the collocation approximate to $w$. From Lemma 5.2 of [15] for $k \geqslant 2 m+n$, we have

$$
\begin{aligned}
\|w-\hat{u}\|_{0, k} & =\left\|S^{-1} S(w-\hat{u})\right\|_{0, k} \\
& \leqslant \gamma_{1}\|S w-S \hat{u}\|_{0, k-n} \\
& \leqslant \gamma_{2}\|w\|_{0, k} \\
& =\gamma_{2}\left\|S^{-1} \hat{v}\right\|_{0, k} \\
& \leqslant \gamma_{3}\|\hat{v}\|_{0, k-n} \\
& =\gamma_{3}\|\hat{v}\|_{0,2 m-1} \\
& \leqslant \gamma_{3}\left[\|v-\hat{v}\|_{0,2 m-1}+\|v\|_{0,2 m-1}\right] \\
& \leqslant \gamma_{3}\|v-\hat{v}\|_{0,2 m-1}+\gamma_{4}\|u\|_{0,2 m+n} .
\end{aligned}
$$

From inequality (6.25) of Theorem 6.5 we have

$$
\|v-\hat{v}\|_{0,2 n} \leqslant \gamma_{5}\|u\|_{0, m+3 n} .
$$

Combining this with (6.39) yields

$$
\|w-\hat{u}\|_{0, k} \leqslant \gamma_{6}\|u\|_{0, m+3 n}
$$

for all $k \geqslant 2 m+n$.

From equations (6.19), (6.26), and (6.27) of Theorem 6.5, and for each $s \in \Delta$ and $j=0,1, \ldots, n-1$, we have

$$
\left|u^{(j)}(s)-\hat{u}^{(j)}(s)\right| \leqslant\left|E_{1 j}(s)\right|+\left|E_{2 j}(s)\right|,
$$

where

$$
\left|E_{1,}(s)\right| \leqslant h^{2 m}\left[\gamma_{7}\|u\|_{0,2 m+2 n}+\gamma_{8}\|u-\hat{u}\|_{0,2 m+n}\right]
$$


and

$$
\left|E_{2 j}(s)\right| \leqslant h^{2 n}\left[\gamma_{9}\|u\|_{0, m+3 n}+\gamma_{10}\|u-\hat{u}\|_{0,3 n}\right] .
$$

From (6.41) with $k \geqslant 2 m+n$

$$
\begin{aligned}
\|u-\hat{u}\|_{0, k} & \leqslant\|u-w\|_{0, k}+\|w-\hat{u}\|_{0, k} \\
& \leqslant\|u-w\|_{0, k}+\gamma_{6}\|u\|_{0, m+3 n} \\
& =\left\|S^{-1}(v-\hat{v})\right\|_{0, k}+\gamma_{6}\|u\|_{0, m+3 n} \\
& \leqslant \gamma_{7}\|v-\hat{v}\|_{0, k-n}+\gamma_{6}\|u\|_{0, m+3 n} .
\end{aligned}
$$

Combining (6.45) with (6.40) for $k=2 m+n$ or $k=3 n$, we have

$$
\|u-\hat{u}\|_{0,2 m+n} \leqslant \gamma_{8}\|u\|_{0, m+3 n}
$$

and

$$
\|u-\hat{u}\|_{0,3 n} \leqslant \gamma_{9}\|u\|_{0, m+3 n} .
$$

Equations (6.42), (6.43), (6.44), (6.46), and (6.47) imply

$$
\left|u^{(j)}(s)-\hat{u}^{(j)}(s)\right| \leqslant \gamma h^{2 m}\|u\|_{0, m+3 n} . \quad \text { Q.E.D. }
$$

Coupling Theorems 6.5 and 6.6 with standard approximation theory yields

THEOREM 6.7. Let $u=L^{-1} f \in C_{\Delta}^{m+n, 2 n}[a, b]$ with $m=n$ or $p<n$. If $m=n$, $m=n-1, m+p \leqslant n$, or $S$ is invertible, then for each $j=0,1, \ldots, \min \{n-$ $1,2 m\}$ :

$$
\left\|u^{(J)}-\hat{u}^{(j)}\right\|_{\infty} \leqslant \gamma h^{2 m-J}\|u\|_{0, m+3 n} .
$$

If $m+p>n$ with $m \neq n, m \neq n-1$, and $\mathscr{R}(S) \neq L^{2}[a, b]$, then for each $j=$ $0,1, \ldots, \min \{n-1,2 m, m+n-p\}$ :

$$
\left\|u^{(J)}-\hat{u}^{(j)}\right\|_{\infty} \leqslant \gamma h^{\min \{2 m, m+n-p\}-J}\|u\|_{0, m+3 n} .
$$

REMARK. The case $m \neq n, m \neq n-1$, and $\mathscr{R}(S) \neq L^{2}[a, b]$ with $m+p>n$ is awkward. We are unable to establish the optimality of (6.49) and its superconvergence analogue. One suspects superconvergence at the rate $h^{2 m}$ and (6.48) should obtain in all cases. The Moore-Penrose generalized inverse of $S$ in Theorem 6.6 is a logical tool for trying to prove this. The mathematics of such an argument is quite intricate and we did not attempt it. 


\section{Acknowledgement}

This work was partially supported by the National Science Foundation under Grants MCS77-02730 and MCS-829-1875.

\section{References}

[1] U. Ascher, J. Christiansen, and R. D. Russell, "A collocation solver for mixed order systems of boundary value problems", Math. Comp. 33 (1979), 659-679.

[2] R. Bellman "A note on an inequality of E. Schmidt", Bull. Amer. Math. Soc. 50 (1944), 734-736.

[3] A. Bracha-Barak, "A factorization procedure for the solution of multidimensional elliptic partial differential equations", SIAM J. Numer. Anal. 11 (1974), 887-893.

[4] J. Cerrutti, "Collocation for systems of ordinary differential equations", Comp. Sci. Tech. Rep. 230, Univ. Wisconsin-Madison, 1974.

[5] C. de Boor and R. Weiss, "Solveblok: A package for solving almost block diagonal linear systems, with applications to spline approximation and the numerical solution of ordinary differential equations", MRC TSR \#1625, Madison, Wisconsin, 1976.

[6] C. de Boor and R. Weiss, "Lobato, A package for the solution of a nonlinear boundary value problem by collocation", to appear.

[7] L. W. Ehrlich, "Solving the biharmonic equation as coupled finite difference equations", SIAM J. Numer. Anal. 8 (1971), 278-287.

[8] L. W. Ehrlich and M. M. Gupta, "Some difference schemes for the biharmonic equation", SIAM J. Numer. Anal. 12 (1975), 773-790.

[9] R. Glowinski and $O$. Pironneau, "Numerical methods for the first biharmonic equation and for the two-dimensional Stokes problem", SIAM Rev. 21 (1979), 167-212.

[10] M. M. Gupta, "Discretization error estimates for certain splitting procedures for solving first biharmonic boundary value problems", SIA M J. Numer. Anal. 12 (1975), 364-377.

[11] E. Houstis, "A collocation method for systems of nonlinear ordinary differential equations", $J$. Math. Anal. Appl. 62 (1978), 24-37.

[12] E. Isaacson and H. B. Keller, Analysis of numerical methods (John Wiley and Sons, New York, 1966).

[13] D. C. Jespersen, "A least squares decomposition method for solving elliptic equations", Math. Comp. 31 (1977), 873-880.

[14] H. B. Keller, "Numerical solution of boundary value problems for ordinary differential equations: Survey and some recent results on difference methods", in Numerical solutions of boundary value problems for ordinary differential equations (ed. A. K. Aziz), (Academic Press, New York, 1975).

[15] J. Locker and P. M. Prenter, "Optimal $L^{2}$ and $L^{\infty}$ error estimates for continuous and discrete least squares methods for boundary value problems", SIAM J. Numer. Anal. 15 (1978), 1151-1160.

[16] J. Locker and P. M. Prenter, "On least squares methods for linear two-point boundary value problems", in Functional analysis methods in numerical analysis (Proc., St. Louis, MO, 1977), (ed. M. Z. Nashed), Lecture Notes in Math. 701 (Springer-Verlag, Berlin-Heidelberg-New York, 1979), 149-168. 
[17] J. Locker and P. M. Prenter, "Regularization with differential operators. I: General theory", $J$. Math. Anal. Appl. 74 (1980), 504-529.

[18] J. Locker and P. M. Prenter, "Regularization with differential operators. II: Weak least squares finite element solutions to first-kind integral equations", SIAM J. Numer. Anal. 17 (1980), 247-267.

[19] J. Locker and P. M. Prenter, "Representors and superconvergence of least squares finite element approximates", Numer. Funct. Anal. Optim., to appear.

[20] M. Z. Nashed (ed.), Generalized inverses and applications (Academic Press, New York, 1976).

[21] R. D. Russell and L. F. Shampine, "A collocation method for boundary value problems", Numer. Math. 19 (1972), 1-28.

[22] P. Sammon, "The discrete least squares method", Math. Comp. 31 (1977), 60-65.

[23] M. Schechter, Principles of functional analysis (Academic Press, New York, 1971).

[24] V. G. Sigillito, "A priori inequalities and approximate solutions of the first boundary value problem for $\Delta^{2} u=f "$, SIAM J. Numer. Anal. 13 (1976), 251-260.

[25] J. Todd (ed.), A survey of numerical analysis (McGraw-Hill, New York, 1962). 